\title{
Hyperuricemia in Early Pregnancy A Marker for Gestational Diabetes Mellitus
}

\author{
Aparna Kappaganthu, Jyoti Sachan, G. Shailaja \\ Dept of Obstetrics and Gynaecology, St Theresa's Hospital. Hyderabad, India
}

\begin{abstract}
Aim: Our study aimed to examine prospectively whether serum uric acid levels (and hyperuricemia) in the first trimester is associated with subsequent development of GDM in a random sample of pregnant women attending Antenatal clinics in a busy General hospital in Hyderabad, India.

Materials and Method: Two hundred and twenty five pregnant women ( $<20$ weeks gestation) were included in the study. Baseline assessments included age, BMI, diet, parity and serum uric acid level. Subjects were reassessed at 24-28 weeks for Gestational Diabetes mellitus (GDM). Data was systematically recorded and analysed to test for associations of various parameters with GDM

Results: The mean baseline serum uric acid level was $2.6 \mathrm{mg} / \mathrm{dL}$. $5.1 \%$ of the subjects developed GDM when assessed at 24-28 weeks. Although within the normal range, serum uric acid level did increase with age, as did the risk of GDM. Diet, socio-economic status and BMI were not associated with an increase of either serum uric acid level or the risk of GDM. The results showed a clear increase in the risk of GDM with increasing serum uric acid levels. A serum uric acid cut-off value 3.4mg/dl had a sensitivity of 90\%, specificity of 95\% and a negative predictive value of $99 \%$ for subsequent development of GDM.

Conclusion: Our study reiterates the association of raised serum uric acid levels in early pregnancy as a risk factor for subsequent development of GDM in an Indian population. A cut off level of $3.4 \mathrm{mg} / \mathrm{dL}$ appears to have good sensitivity and specificity in identifying those patients who are most likely to develop GDM later in pregnancy. This, if replicated and confirmed, can have important therapeutic implications in helping identify and manage GDM early, and thus prevent adverse maternal and foetal complications.
\end{abstract}

\section{Introduction}

Uric acid is the final oxidation product of purine catabolism. Recent literature suggests a bidirectional causal relationship between hyperuricemia and insulin resistance ( $\mathrm{Li}$, et al 2013). In fact, hyperuricemia has been found to be a marker and predictor for future development of diabetes and the metabolic syndrome (Borges, et al., 2010, Choi and Ford, 2007, Krishnan, et al., 2012). Sex differences have been noted in serum uric acid levels, with hyperuricemic women having a poorer cardiovascular outcomes than men (Borges et al, 2010).

Lately, researchers have focused upon the effects and associations of serum uric acid and hyperuricemia amongst pregnant women. Serum uric acid levels have been reported to be significantly lower during early and middle pregnancy, as compared to age-matched female controls (Boyle, et al, 1966). Laughon et al (2008) from the US found that hyperuricemia was a dose-dependent risk factor for the development of Gestational diabetes mellitus (GDM) amongst pregnant women. GDM is a challenging problem in pregnancy due to the maternal and foetal complications that it causes. An Iranian study by Amini et al (2014) also found early maternal hyperuricemia to be associated with various neonatal complications (including preterm deliveries and IVH).

The reviewed literature suggests hyperuricemia to be an emerging risk factor for GDM and adverse pregnancy outcomes amongst various populations- but particularly so amongst Asians. It is reported these ethnic differences may translate into differential risks for GDM across ethnicities (DeBoer, et al, 2012, Carolan, et al 2012). In our search, we could not find any Indian studies that looked the association of hyperuricemia with GDM.

Our study aimed to examine prospectively whether serum uric acid levels (and hyperuricemia) in the first trimester is associated with subsequent development of GDM in a random sample of pregnant women attending Antenatal clinics in a busy General hospital in Hyderabad, India.

\section{Methods}

The study was conducted as part of a postgraduate dissertation submitted to the National Board of Examinations. It was conducted in the Obstetrics and Gynaecology (OBG) department of St. Theresa's hospital in Hyderabad, India. This is a large teaching general hospital situated within a busy urban locality of the city. The OBG department consists of Consultants and postgraduate trainees who run subsidised antenatal, delivery 
and postnatal services, and cater to patients hailing from a predominantly middle and lower socio-economic background.

The study was conducted over a period of 1-year from May 2013. The sample size was ascertained after a power calculation in consultation with the department's statistician. The study sample was drawn from amongst pregnant women who had registered with the antenatal clinic of the hospitals.

A convenience sample of 225 consenting subjects was recruited for the study. Patients with preexisting diabetes mellitus, hypertension, gout, connective tissue disorders or on medications causing hyperuricemia (eg, pyrazinamide, ethambutol, levodopa and theophylline) were excluded. Eleven subjects were lost to follow up. This study reports on findings of the remaining 214 subjects for whom data was available.

At the initial antenatal assessment (which included a detailed history and an examination), the following details were also collated for the purposes of this study: age, social class, dietary habits (vegetarian or non-vegetarian), parity, weight, height, BMI, baseline serum uric acid and a fasting blood sugar.

All participating subjects were reviewed at around 24-28 weeks of gestation, when an Oral glucose tolerance test (OGTT) was done. The subjects were followed up till term for maternal and foetal outcome, as part of the antenatal service.

Data was systematically recorded in a proforma and analysed descriptively using t-tests, ANOVA and chi-squared tests (as appropriate) for statistical significance. A ROC analysis was done to identify a cut-off for serum uric acid levels to predict subsequent GDM.

\section{Results}

Two hundred and twenty five subjects were enrolled in the study. Of these, 11 were lost to follow-up. The mean age of the sample was 23.6 years $(\mathrm{SD}=2.9)$. Ninety-four percent of the subjects described themselves as hailing from a middle and lower socio-economic background, and $81 \%$ described their diet as being nonvegetarian.. The majority was nulliparous $(47 \%)$ or primiparous $(38 \%)$. Baseline BMI (body mass index) was calculated using weight and height data. The majority (61\%) had a BMI between 18.5 and 24.9, with the mean BMI being 22.0 ( $\mathrm{SD}=3.9)$.

The mean serum uric acid level in the entire sample was $2.6 \mathrm{mg} / \mathrm{dL} .11$ of the 214 subjects developed GDM on follow up (5.1\%). Table 1 shows that the mean serum uric acid levels increase with age and this was found to be statistically significant $(\mathrm{p}<0.05)$. The results also showed that a significantly higher proportion of older subjects developed GDM compared to younger subjects $(\mathrm{p}<0.05)$.

Table:1 Serum Uric acid levels and GDM in different age groups.

\begin{tabular}{|l|l|l|l|l|}
\hline \multirow{2}{*}{ Age (in years) } & Uric acid concentration $(\mathbf{m g} / \mathbf{d l})$ & \multicolumn{3}{l|}{ OGTT status } \\
\cline { 2 - 5 } & Range & Mean \pm SD & Normal & Abnormal (GDM) \\
\hline$\leq 20(\mathrm{n}=43)$ & $1.6-4.2$ & $2.49 \pm 0.6$ & 39 & 4 \\
\hline $21-25(\mathrm{n}=120)$ & $1.7-4.0$ & $2.59 \pm 0.4$ & 118 & 2 \\
\hline $26-30(\mathrm{n}=40)$ & $1.8-4.2$ & $2.77 \pm 0.6$ & 37 & 3 \\
\hline$>30(\mathrm{n}=11)$ & $1.9-4.1$ & $2.98 \pm 0.7$ & 9 & 2 \\
\hline
\end{tabular}

The results also showed that the mean uric acid levels were significantly higher in multiparous women as compared to nulliparous and primiparous women ( $<<0.05)$, but the proportion of patients with GDM did not increase with parity. Similarly, the serum uric acid levels and prevalence of GDM did not differ with diet, BMI and socio-economic status.

In order to answer the study question, the data was analysed to explore the association of serum uric acid levels with the development of GDM (Table 2). The results show that a significantly higher proportion of women with higher serum uric acid levels developed GDM, compared to those with lower serum uric acid levels $(\mathrm{p}<0.05)$.

Table 2-Correlation of Serum uric acid level with OGTT status

\begin{tabular}{|l|l|l|l|}
\hline $\begin{array}{l}\text { Serum uric acid } \\
\text { concentration } \\
\text { (mg/dl) }\end{array}$ & \multirow{2}{*}{ No. of subjects } & \multicolumn{2}{|l|}{ OGTT status } \\
\cline { 3 - 4 } & & $\begin{array}{l}\text { Normal } \\
\text { (non GDM) }\end{array}$ & $\begin{array}{l}\text { abnormal } \\
\text { (GDM) }\end{array}$ \\
\hline $1.0-2.0$ & 42 & 42 & 0 \\
\hline $2.1-3.0$ & 139 & 138 & $1(0.7 \%)$ \\
\hline $3.1-3.9$ & 26 & 22 & $4(15 \%)$ \\
\hline$\geq 4$ & 7 & 1 & $6(85 \%)$ \\
\hline
\end{tabular}

OGTT: Oral Glucose Tolerance test, GDM: Gestational Diabetes Mellitus.

Finally, a ROC analysis was done to ascertain a suitable serum uric acid cut-off so as to be suggested as a marker for subsequent development of GDM. 
Figure 1: ROC curve for serum uric acid in relation to an outcome of GDM

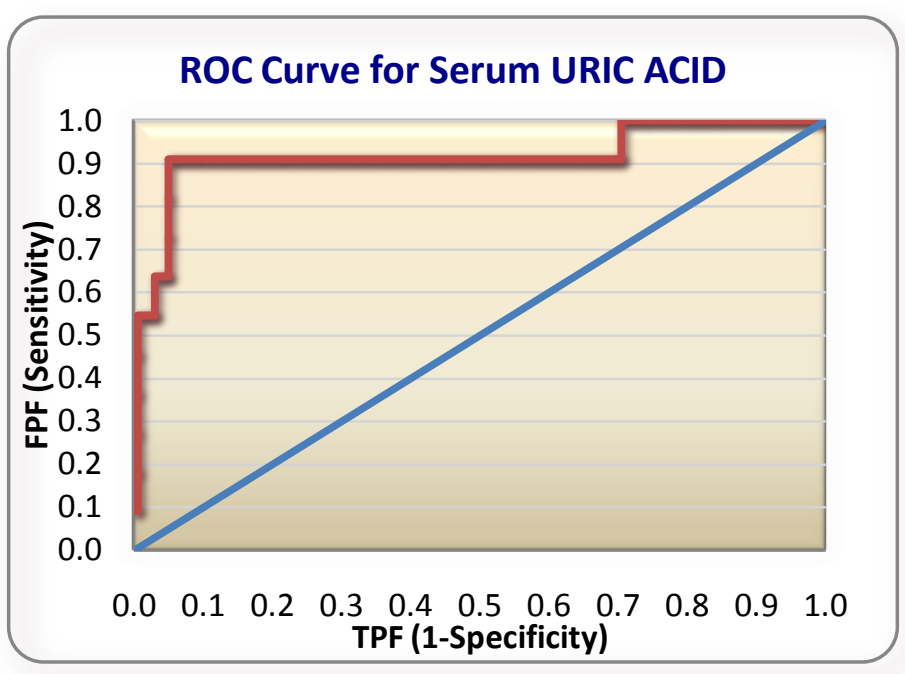

Area under the curve- 0.9176 , Standard error- 0.0904

A cut-off Serum uric acid level of $3.4 \mathrm{mg} / \mathrm{dL}$ was found to have a sensitivity of $90 \%$, specificity of $95 \%$, a positive predictive value of $50 \%$ and a negative predictive value of $99 \%$ for the development of GDM. The distribution of cases that developed GDM at a cut-off uric acid level of $3.4 \mathrm{mg} / \mathrm{dL}$ is shown in table 3

Table 3- Distribution of GDM cases using serum uric acid cut-off of $3.4 \mathrm{mg} / \mathrm{dl}$

\begin{tabular}{|l|l|l|l|}
\hline Uric acid (mg/dl) & GDM & Non GDM & Total \\
\hline$<3.4$ & 1 & 191 & 192 \\
\hline$\geq 3.4$ & 10 & 12 & $\mathbf{2 2}$ \\
\hline Total & 11 & 203 & 214 \\
\hline
\end{tabular}

\section{Discussion}

This study was conducted in an urban General hospital antenatal service setting in South India. The socio-economic, demographic and dietary charcteristics of our subjects are quite typical of patients encountered in most antenatal services in this part of the world- as such, the findings are likely to be generalisable across the region. The sample size of 225 subjects was estimated using a power calculation based on the prevalence of GDM, and was guided by the department statistician, This (and the low drop-out rate) are felt to be strengths of our study. In terms of potential limitations, our study did not specifically loook at some of the other reported associations of hyperuricemia in literature- like metabolic syndrome, and maternal and fetal outcomes- which would have helped clarify the potential impact of hyperuricemia further.

The mean serum uric acid level across the sample was $2.6 \mathrm{mg} / \mathrm{dL}$ and $5.1 \%$ of the subjects developed GDM on follow up. Both these parameters are well within the normal range as described in literature. Amongst the background variables examined, only age seemed to be associated with an increase in serum uric acid levels and the development of GDM. This finding is in keeping with previous studies that have reported increasing prevalence of GDM, hyperuricemia and serum uric acid levels with age amongst women (Qiu, et al 2013, Carolan, et al, 2012). Although there were some indications that subjects on a solely vegetarian diet are likely to have lower uric acid levels than those on other diets, sub-group analyses have found this effect to be nonsignificant (Tsai, et al, 2012), and this is reflected in our findings too. Similarly, although some studies have reported parity to be a risk factor for GDM (Al Rowaily, et al, 2010), our study did not reflect this findingperhaps due to the confounding effect of age on the same outcome.

The results from our study suggest that increasing serum uric acid level was associated with a higher risk of developing GDM. This finding is in keeping with Laughon's 2008 study which also found a dose-related increase in GDM with increases in uric acid levels. It suggests that raised serum uric acid could serve as a marker for subsequent development of GDM. In order to explore this further, we conducted a Reciever operating characteristics analysis to ascertain an appropriate cut-off for serum uric acid level that might best predict GDM. This is in line with Borges, et al's suggestion that hyperuricemia could be used as an efffective marker for later development of metabolic syndrome and Diabetes. However, none of the subjects in our study were actually hyperuricemic (defined as $>6 \mathrm{mg} / \mathrm{dL}$ ). This notwithstanding increases in serum uric acid levels was still associated with a higher risk of developing GDM. A cut off of $3.4 \mathrm{mg} / \mathrm{dL}$ seemed to achieve a good balance of sensitivity and specificity in this population. However, the usefulness and predictive value of this cut-off needs to be piloted and tested in larger sample sizes before it can be widely recommended. 


\section{Conclusions}

Our study reiterates the association of raised serum uric acid levels in early pregnancy as a risk factor for subsequent development of GDM in an Indian population. A cut off level of $3.4 \mathrm{mg} / \mathrm{dL}$ appears to have good sensitivity and specificity in identifying those patients who are most likely to develop GDM later in pregnancy. This, if replicated and confirmed, can have important therapeutic implications in helping identify and manage GDM early, and thus prevent adverse maternal and foetal complications.

\section{References}

[1]. Al-Rowaily MA, Abolfotouh MA.. Predictors of gestational diabetes mellitus in a high-parity community in Saudi Arabia. East Mediterr Health J. 2010 Jun;16(6):636-41.

[2]. Amini E, Sheikh M, Hantoushzadeh S, Shariat M, Abdollahi A and Kashanian M. Maternal hyperuricemia in normotensive singleton pregnancy, a prenatal finding with continuous perinatal and postnatal effects, a prospective cohort study. BMC Pregnancy and Childbirth 2014, 14:104

[3]. Borges RL, Ribeiro AB, Zanella MT, Batista MC. Uric acid as a factor in the metabolic syndrome. Curr Hypertens Rep. 2010 Apr;12(2):113-9.

[4]. Boyle JA, Campbell, Duncan AM, Greig WR and Buchanan WW. Serum uric acid levels in normal pregnancy with observations on the renal excretion of urate in pregnancy. J Clin Pathol. Sep 1966; 19(5): 501-503.

[5]. Carolan M, Davey MA, Biro MA, Kealy M.. Maternal age, ethnicity and gestational diabetes mellitus. Midwifery. 2012 Dec;28(6):778-83.

[6]. Choi HK, Ford ES. Prevalence of the metabolic syndrome in individuals with hyperuricemia. Am J Med. 2007 May;120(5):442-7.

[7]. DeBoer MD, Dong L, Gurka MJ. Racial/ethnic and sex differences in the relationship between uric acid and metabolic syndrome in adolescents: an analysis of National Health and Nutrition Survey 1999-2006. Metabolism. 2012 Apr;61(4):554-61.

[8]. Krishnan E, Pandya BJ, Chung L, Hariri A, DabbousDisclosures O.Hyperuricemia in Young Adults and Risk of Insulin Resistance, Prediabetes, and Diabetes: A 15-Year Follow-up Study Am J Epidemiol. 2012;176(2):108-116.

[9]. Laughon SK, Catov J, Provins T, Roberts, Gandley R. First trimester hyperuricemia predicts development of gestational diabetes. American Journal of Obstetrics \& Gynecology 2008: 199, 6, Supplement A, S124.

[10]. Li C, Hsieh MC, Chang SJ. Metabolic syndrome, diabetes, and hyperuricemia. Curr Opin Rheumatol. 2013 Mar;25(2):210-6.

[11]. Qiu L, Cheng X, Wu J, Liu J, Xu T, Ding H, Liu Y, Ge Z, Wang Y, Han H, Liu J and Zhu G. Prevalence of hyperuricemia and its related risk factors in healthy adults from Northern and Northeastern Chinese provinces. BMC Public Health 2013, 13:664

[12]. Tsai YT, Liu JP, Tu YK, Lee MS, Chen PR, Hsu HC, Chen MF, Chien KL.. Relationship between dietary patterns and serum uric acid concentrations among ethnic Chinese adults in Taiwan. Asia Pac J Clin Nutr. 2012;21(2):263-70. 